\title{
SPUTTERING IN INTERSTELLAR SHOCKS: A MODEL FOR HEAVY ELEMENT DEPLETION
}

\author{
Michael J. BARLOW \\ Joint Institute for Laboratory Astrophysics, University of Colorado and National Bureau of Standards, Boulder
}

AND

JOSEPH SILK*

Institute for Advanced Study, Princeton

Received 1976 August 20; revised 1976 October 12

\begin{abstract}
High-velocity $\left(~ \gtrsim 100 \mathrm{~km} \mathrm{~s}^{-1}\right.$ ) shock fronts are found to provide an environment where grains can be destroyed by thermal sputtering. Application is made to supernova remnants. Sputtering of refractory grains in shocks associated with high-velocity clouds leads to cosmic abundances of heavy elements, and is followed by deceleration of the high-velocity gas and dilution with ambient depleted matter. A correlation is predicted of depletion with systematic velocity. We propose that there is a residual underlying depletion associated with refractory grain formation, and a subsequent trapping of gas-phase metallic species by adsorption. Sputtering of adsorbed monolayers in intermediate velocity shocks $\left(\leq 50 \mathrm{~km} \mathrm{~s}^{-1}\right)$ can account for a range of depletions in diffuse interstellar matter.
\end{abstract}

Subject headings: interstellar: matter - shock waves

\section{INTRODUCTION}

Optical and ultraviolet observations of interstellar absorption lines have revealed that systematic patterns exist in the depletion factors observed for many heavy elements. The greatest depletions always seem to be manifested by the metallic elements, whereas nonmetallic elements are usually only lightly depleted. The generally accepted explanation for the observed depletions is that the missing atoms are locked up in interstellar grains.

However, although refractory grain formation may account for an underlying and constant depletion of the metallic elements (Field 1974), it cannot explain the large variations observed in the depletion factors of the same elements along lines of sight where the physical conditions vary widely (cf. Hobbs 1976; Jenkins, Silk, and Wallerstein 1976; Shull and York 1977). The observational data evidently require a mechanism whereby the heavy elements in grains can be returned to the gas, giving variable depletion factors in diffuse clouds. This requirement must take account of the likelihood that refractory grains, such as graphite or silicates, are the predominant grain constituent in such regions.

Two sputtering mechanisms that utilize interstellar shocks are described below. High-velocity shocks (possibly associated with old supernova remnants) provide a means of destroying grains by thermal sputtering ( $\S \mathrm{II}$ ), and lead to a predicted dependence of depletion on gas velocity ( $\$$ III). Adsorbed monolayers of metallic species can also be sputtered in intermediate-velocity shocks, and may account for a range of depletions in the diffuse interstellar medium $(\S I V)$.

* John Simon Guggenheim Foundation Fellow 1975/1976; normally at University of California, Berkeley.

\section{SPUTTERING OF GRAINS IN HIGH-} VELOCITY SHOCKS

Sputtering rates appropriate to interstellar grains have recently been reexamined by Barlow $(1975,1977)$. The use of experimental data to obtain empirical sputtering-yield formulae and sputtering thresholds $E_{\text {th }}$ of different materials leads to sputtering rates that are considerably less than those derived previously by Aannestad (1973). Only a brief summary is given here of the results of Barlow's calculations.

The rate of destruction of a spherical grain of radius $a$ and density $p_{s}$ by thermal sputtering in a gas of temperature $T$ can be expressed as

$$
\frac{d a}{d t}=\sum_{i} X_{i} m_{s} \rho_{s}^{-1} S_{i}\left(\frac{k T}{2 \pi m_{i}}\right)^{\mathrm{i} / 2} f\left(\frac{E_{t h}}{k T}\right) n,
$$

where $m_{s}$ is the mass of the target particle, $m_{i}$ is the mass of the incident ion, $X_{2}$ is the fractional abundance of the incident ions, the sputtering constants $S_{i}$ are obtained by fitting experimental data, and the function $f\left(E_{\text {th }} / k T\right)$ is obtained by integrating an empirical expression for the sputtering yield per ion over a Maxwellian velocity distribution. It is convenient to introduce the characteristic sputtering time $t_{s}=a(d a / d t)^{-1}$ which varies inversely with total particle density $n$. We have evaluated $n t_{s}$ as a function of $T$ for several grain materials (Fig. 1). Helium nuclei make the major contribution to the sputtering rates, protons and heavier nuclei yielding $30 \%$ and $10 \%$ respectively. Comparison with the cooling time scale $t_{c}$ (also inversely proportional to $n$ ) reveals that only at $T \geqslant 3 \times 10^{5} \mathrm{~K}$ does $t_{c}$ exceed $t_{s}$ for ice grains of radius $a=\widetilde{0} .1 \mu \mathrm{m}$. Since $t_{c}$ determines how long any given volume element of shocked gas 
stays hot (at the postshock temperature), we can infer minimum shock velocities for sputtering to occur from the relation (valid for strong shocks) $v^{2}=16 k T(3 \mu)^{-1}$, where $\mu$ is the mean mass per newly shocked particle and $v$ is the shock velocity. Thus ice grains are effectively destroyed in interstellar shocks where $v \gtrsim 100 \mathrm{~km}$ $\mathrm{s}^{-1}$. Of greater interest, however, is the sputtering of refractory grains. Grain radii of $0.03 \mu \mathrm{m}$ were adopted in all refractory grain calculations, and sputtering rates were calculated including the effects of kinetic grain charging (photoelectric effects being generally negligible away from hot stars). Shock velocities of $v \geq 200 \mathrm{~km} \mathrm{~s}^{-1}$ are required to effectively destroy silicate $\left(\mathrm{MgSiO}_{3}\right)$ grains, and $v \gtrsim 300 \mathrm{~km} \mathrm{~s}^{-1}$ will sputter even the most refractory grain material, graphite. Note that the grain slowing-down time is less than the cooling time for shocks at $\gtrsim 100 \mathrm{~km} \mathrm{~s}^{-1}$, although, in slower shocks, sputtering due to relative motion of grains and gas can continue after completion of cooling.

Comparison with $t_{c}$ is appropriate only if cooling occurs. This requires a minimum hydrogen column density $N=\frac{1}{4} v n t_{c}(n, T)$ in the shocked gas, amounting to $N \approx 10^{18} \mathrm{~cm}^{-2}$ if $v=100 \mathrm{~km} \mathrm{~s}^{-1}$ and $N \approx 10^{20} \mathrm{~cm}^{-2}$ if $v=300 \mathrm{~km} \mathrm{~s}^{-1}$. For higher shock velocities, the shock

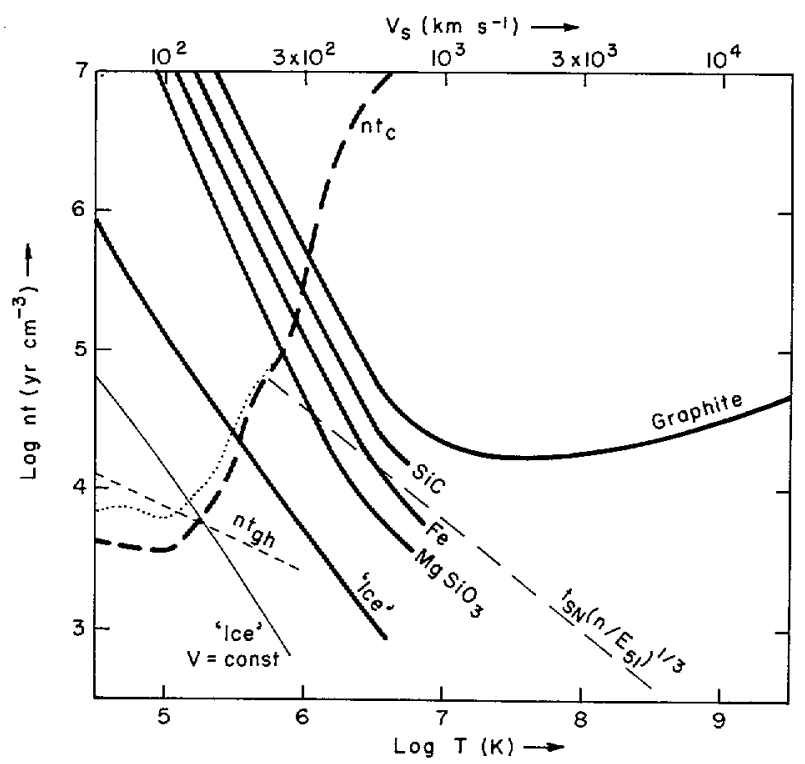

Fig. 1.- Variation of $n t_{s}$ with $T$ (lower abscissa) and $v_{s}$ (upper abscissa) for several materials. Curves labeled "ice" were calculated for a sublimation energy of $0.1 \mathrm{eV}$ and for a grain radius $a=0.1 \mu \mathrm{m}$. For all other materials, we set $a=0.03 \mu \mathrm{m}$. Curve labeled " $v=$ const." refers to ice grains moving at constant velocity $v_{8}$ relative to gas at temperature $T$; all other sputtering curves refer to grains at rest. The effect of grain charge was included. Graphite data are from Burke and Silk (1974). Also shown are the cooling times (labeled $n t_{c}$ ) for a gas mixture of cosmic abundances, both for equilibrium cooling (dashed curve) and for time-dependent cooling from a temperature of $10^{6} \mathrm{~K}$ (dotted curve) (data from Shapiro and Moore 1976). The lifetime of a spherical adiabatic blast wave propagating through a uniform gas of density $n / 4$ is labeled $t_{s n}\left(n / \mathrm{E}_{51}\right)^{1 / 3}$, where $E_{51}$ is the initial blast wave energy in units of $10^{51} \mathrm{ergs}$ and the slowing-down time scale of uncharged grains by encounters with gas atoms is labeled $n t_{g h}$. interaction with typical diffuse clouds $\left(N \leq 3 \times 10^{20}\right.$ $\mathrm{cm}^{-2}$ ) is likely to be adiabatic, and other considerations then apply.

A plausible environment for these high-velocity shocks is in the vicinity of a supernova remnant, where interstellar clouds can be accelerated to considerable velocities. A well-studied example of such clouds at optical and ultraviolet wavelengths occurs in the vicinity of the Vela supernova remnant; the observed depletions will be discussed below. Here we emphasize that in the adiabatic regime there is a critical density for the ambient medium into which a supernova remnant is expanding, below which sputtering will not occur. This arises because the lifetime of an adiabatically expanding supernova remnant propagating into a uniform medium of density $n_{0}$, decelerating according to the Sedov-Taylor blast wave model, is proportional to $\left(E / n_{0}\right)^{1 / 3}$, where $E$ is the blast-wave energy. From Figure 1, it is evident that the sputtering of graphite grains peaks at $\sim 500 \mathrm{~km} \mathrm{~s}^{-1}$, and destruction occurs only if $n_{0} \gtrsim 10 \mathrm{~cm}^{-3}$. Silicate grains survive if $n_{0} \leq$ $0.1 \mathrm{~cm}^{-3} \cdot{ }^{1}$ Any primordial grains produced in the supernova ejecta that have survived the early stages of the supernova explosion are therefore likely to avoid further destruction by sputtering provided they have not been swept up by dense clouds encountered by the remnant in its early adiabatic phase.

Although refractory grains are destroyed only in shock fronts at $v \gtrsim 200 \mathrm{~km} \mathrm{~s}^{-1}$, we now argue that lower-velocity gas may also be enriched as a result of dilution and deceleration of the high-velocity gas.

\section{ASTROPHYSICAL IMPLICATIONS}

Strong evidence for complete grain destruction in high-velocity shocks comes from the data of Jenkins, Silk, and Wallerstein (1976), who find that Fe and Si probably attain their solar abundance level in components at $-90 \mathrm{~km} \mathrm{~s}^{-1}$ and $-180 \mathrm{~km} \mathrm{~s}^{-1}$ toward HD 75821 and HD 74455, whereas these elements are depleted by a large factor in low-velocity gas along these lines of sight.

Gas at intermediate velocities has been studied by Shull, Hobbs, and York (1976), toward $\mu$ Col, HD 28597, and HD 50876, and reveals lower depletion of $\mathrm{Fe}$ and $\mathrm{Si}$ than is found in the low-velocity gas. This gas may have originated at a higher velocity, having been accelerated by supernova remnants and subsequently decelerated according to the snowplow model, as has been proposed by Siluk and Silk (1974) in their study of high-velocity $\mathrm{Na}$ and $\mathrm{Ca}$ components. The high-velocity gas approximately conserves momentum as it sweeps up depleted ambient matter; since the mass of swept-up matter is proportional to $v^{-1}$, one would predict that the fractional depletion $\delta$ of any given heavy element locked up in refractory grains must satisfy

$$
\delta \approx v / v_{\mathrm{sp}}\left(v \leq v_{\mathrm{sp}}\right) ; \quad \delta \approx 1\left(v \geq v_{\mathrm{sp}}\right),
$$

${ }^{1}$ It is of interest to note that the mean lifetime of interstellar silicate grains with regard to destruction in supernova remnants is $\sim 3 \times 10^{9} \mathrm{yr}$, if conventional estimates for supernova frequency and mean gas density are adopted. 
where $v_{\mathrm{sp}} \approx 200 \mathrm{~km} \mathrm{~s}^{-1}$ is the minimum shock velocity 1 at which effective refractory grain destruction can occur. Relation (1) is valid for velocities $v \geq \delta_{0} v_{\mathrm{sp}}$, where $\delta_{0}$ is the residual depletion of the ambient gas due to refractory grain membership. Comparison of data toward reddened and unreddened stars (York 1977) suggests that species possess an underlying and relatively uniform depletion which varies from element to element, but for $\mathrm{Si}$ and $\mathrm{Fe}$ may be $\delta_{0} \approx 0.1$. We remark further on the significance of this result below; here we note that formula (1) should be valid for $v \gtrsim 20 \mathrm{~km} \mathrm{~s}^{-1}$.

Observational data on depletion as a function of radial velocity for a number of interstellar absorption components toward relatively unreddened stars is shown in Figure 2 for $\mathrm{Fe}$ and $\mathrm{Si}$, over the velocity range (relative to the local standard of rest) $10 \mathrm{~km} \mathrm{~s}^{-1} \leq\left|v_{r}\right| \leq$ $180 \mathrm{~km} \mathrm{~s}^{-1}$. Iron reveals a continuous trend, ranging from $\delta \approx 0.02$ in the $10 \mathrm{~km} \mathrm{~s}^{-1}$ component $^{2}$ of HD 28497 to approximately solar abundance in HD 75821 and HD 74455. On the other hand, $S$ (not displayed in Fig. 2) appears to have approximately cosmic abundances at all velocities, consistent with its not being significantly depleted by grain membership.

We compare the Fe and Si depletions in Figure 2 with

${ }^{2}$ This component shows $\mathrm{Fe}$ and $\mathrm{Si}$ depletions characteristic of low-velocity gas toward unreddened stars: the average depletions for seven components consisting of low-velocity gas ( $\left|v_{\mathrm{LSR}}\right|<$ $10 \mathrm{~km} \mathrm{~s}^{-1}$ ) toward the stars utilized in Figure 1, together with $\alpha$ Vir (York and Kinahan 1976) and $\gamma$ Ara (Morton and Hu 1975), are $\log \delta(\mathrm{Fe})=-1.5 \pm 0.1(\mathrm{rms})$ and $\log \delta(\mathrm{Si})=-1.1 \pm 0.3$ (rms).

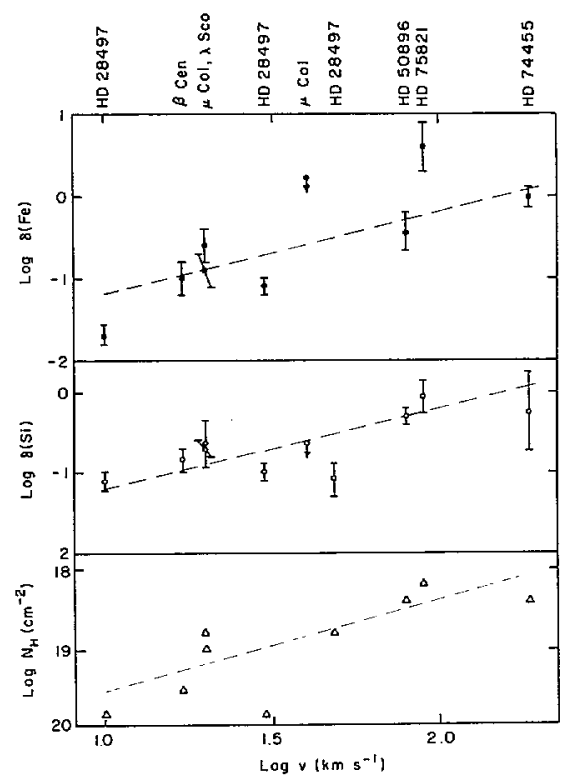

FIG. 2.-Variation of Fe and $\mathrm{Si}$ depletion and hydrogen column density with velocity. Data are shown for components with $\left|v_{\mathrm{LSR}}\right| \geq 10 \mathrm{~km} \mathrm{~s}^{-1}$ in the following stars: HD 74455 and $\mathrm{HD}$ 75821 (Jenkins et al. 1976); HD 50896, HD 28497, and $\mu \mathrm{Col}$ (Shull et al. 1977); $\lambda$ Sco (York 1975, 1977); and $\beta$ Cen (York 1977; Bohlin 1975). The dashed lines show the $v$ proportionality prcdicted by the snowplow model (see text): the normalization is chosen to give an approximate fit to the data points. the $v^{+1}$ dependence expected if the depletions are produced primarily in shocks at $v \approx v_{\mathrm{sp}}$. Also shown are the column densities $N_{\mathrm{H}}$ of the various components. The available data for $\mathrm{Fe}$ and possibly also for $\mathrm{Si}$ are consistent with the occurrence of refractory grain destruction in high-velocity shocks, attaining cosmic abundances at the highest observed velocities. The increase of $V_{\mathrm{H}}$ with decreasing $v$ is also consistent with a snowplow model, in which dilution with depleted matter accounts for the greater depletions found at intermediate velocities.

Siluk and Silk (1974) have also found that $\mathrm{Ca}$ is enhanced relative to $\mathrm{Na}$ in components at $\left|v_{r}\right| \geq 20 \mathrm{~km} \mathrm{~s}^{-1}$ which can be interpreted as decelerated supernova remnants. The preceding model is capable of accounting for the variations in $\mathrm{Ca} / \mathrm{Na}$ with component velocity if $\mathrm{Ca}$ is preferentially locked up (relative to $\mathrm{Na}$ ) in grains.

Apparently, whereas $\mathrm{Fe}$ is significantly enhanced at intermediate velocities compared with the low-velocity gas, the Si depletion is approximately constant in components below $\sim 70 \mathrm{~km} \mathrm{~s}^{-1}$. It is difficult to see how refractory grain destruction could distinguish between $\mathrm{Fe}$ and $\mathrm{Si}$. We therefore propose that at least two depletion mechanisms may be operative, one being refractory grain membership and the other being adsorption onto grain surfaces. The latter mechanism, as we now demonstrate, preferentially depletes metals (e.g., Fe but not Si).

\section{SFUTTERING OF ADSORBED MONOLAYERS}

A low-energy atom impinging onto the surface of a grain will be adsorbed. For the case of graphite grains (which are of a metallic nature), atoms will be chemisorbed with a binding energy of several $\mathrm{eV}$ whereas dielectric silicate (or ice) grains (which are insulators) will only physically adsorb atoms, with a binding energy that is typically an order of magnitude less. Subsequently the adsorbed atoms rapidly recombine with hydrogen atoms which are migrating on the surface of the grain. Each recombination with an $\mathrm{H}$ atom liberates energy and simultaneously weakens the bond to the surface. If the total recombination energy liberated in forming a saturated hydride molecule exceeds the sum of the adsorption binding energies of the constituent atoms, then the hydride can be ejected from the surface; if the reverse holds, the heavy atom will be trapped on the surface, unable to escape from its potential well.

Physically adsorbing grain surfaces have been examined by Barlow (1974), who concluded, using the data of Aannestad (1972) on physical adsorption binding energies of various elements, that only $\mathrm{Ca}$ and $\mathrm{Ti}$ (due to their large polarizabilities) would be trapped on the surfaces of grains such as silicates. Chemisorption binding energies on graphite have been calculated for many elements and compared to the total recombination energy liberated in hydride formation by Barlow (1975, 1976). The ionization state of the incoming atom plays no significant role in depletion, and it is found that metallic elements are trapped, whereas nonmetallic atoms can escape. If other metallic grains such as magnetite exist, they will also deplete metals in a manner similar to graphite. 
Metallic elements in the interstellar medium will thus undergo progressive depletion onto grains until conditions suitable for returning them to the gas phase arise. The existence of experimental data and a simple theoretical interpretation on sputtering of chemisorbed monolayers (Winters and Sigmund 1974) has enabled us to quantitatively examine the sputtering in shock fronts of many different species of atoms chemisorbed onto graphite grains (Barlow and Silk 1976; Barlow 1977). This sputtering mechanism, specific to chemisorbed atoms, is found to be much more efficient for a given incident energy than the usual physical sputtering of solids.

Using theoretical expressions given by Winters and Sigmund for estimating the sputtering yield as a function of adsorption energy and incoming ion energy and mass, we conclude that shocks as slow as $\sim 20 \mathrm{~km} \mathrm{~s}^{-1}$ can sputter $\mathrm{Li}, \mathrm{Na}, \mathrm{Mg}$, and $\mathrm{K}$, whereas significantly faster shocks $\left(\sim 40 \mathrm{~km} \mathrm{~s}^{-1}\right)$ are required to sputter Ti, $\mathrm{Fe}$, and $\mathrm{Mn}$. Calcium and $\mathrm{Al}$ are sputtered above about $\sim 30 \mathrm{~km} \mathrm{~s}^{-1}$. Neither Ar, which does not recombine but is easily removed above $\sim 5 \mathrm{~km} \mathrm{~s}^{-1}$, nor $\mathrm{Si}$, which behaves like a nonmetal and undergoes ejection as a saturated hydride, is expected to be depleted by chemisorption.

A significant amount of heavy elements can be contained in surface monolayers. We estimate that the number of surface sites available on small graphite grains of radius $\sim 0.02 \mu \mathrm{m}$ (Gilra 1972) can accommodate a total abundance relative to hydrogen of $\sim 3 \times$ $10^{-6}$. Chemisorption onto graphite grains therefore cannot cause the majority of the depletion observed for $\mathrm{Fe}$ and $\mathrm{Mg}$, for which refractory grain membership must therefore be responsible, although it could be important for $\mathrm{Na}, \mathrm{Ca}, \mathrm{K}$, and $\mathrm{Ti}$. Although no relevant experimental data are available on the sputtering of physically adsorbed monolayers, it seems plausible that such layers, notably of $\mathrm{Ca}$ and $\mathrm{Ti}$, should also be easily removed in shocks of relatively low velocity.

Shock sputtering of adsorbed monolayers may actually account for the observed differential depletion of atomic species in the general low-velocity gas. Consider a model in which $\mathrm{Fe}, \mathrm{Si}, \mathrm{Na}, \mathrm{Ca}$, etc., have an underlying and uniform depletion factor $\delta_{0}$ produced by membership in refractory grains (cf. Field 1974). Grains will adsorb monolayers of metals in diffuse clouds where any ice mantles are likely to be photodesorbed (Barlow 1975), leading to further depletion of these species. Now the observed frequency of intermediate-velocity clouds (Siluk and Silk 1974) suggests that a typical intercloud atom will have been swept up in a shock of $v \gtrsim 40 \mathrm{~km}$ $\mathrm{s}^{-1}$ over the lifetime $\left(\sim 10^{8} \mathrm{yr}\right)$ of the intercloud medium, and grain surfaces will have been swept clean of adsorbed monolayers. In clouds, however, depletion occurs and metallic species will tend to remain adsorbed over the cloud lifetime. The species with the lowest adsorption binding energies $(\mathrm{Na}, \mathrm{Li}, \mathrm{K}, \mathrm{Mg}$ ) will be less depleted than more tightly bound species $(\mathrm{Ca}, \mathrm{Fe}, \mathrm{Ti})$ since these monolayers will have been more recently removed by low-velocity shock sputtering. Thus we can account for the generally lower depletions observed toward unreddened stars (Hobbs 1976; York 1977), and may moreover be able to understand the range of depletions observed in diffuse clouds, where the depletion of any given species will depend on its adsorption energy: The latter statement necessarily remains qualitative in view of the need for a far more specific grain model before any predictions are feasible; however, we emphasize that if, for example, $\delta_{0} \approx 0.1-0.3$, metallic atoms with a gas-phase abundance relative to hydrogen of less than $10^{-5}$ can be significantly depleted by adsorbed monolayers. Thus the variations in $\mathrm{Fe}$ depletion between reddened and unreddened stars could possibly be understood in terms of this model, although we consider the mechanism likely to be more significant for rarer species $(\mathrm{Ca}, \mathrm{Na}, \mathrm{Ti}, \mathrm{K})$. This mechanism provides an additional means of explaining the $\mathrm{Ca} / \mathrm{Na}$ enhancement found in components moving at $\gtrsim 20 \mathrm{~km} \mathrm{~s}^{-1}$. High-latitude gas also appears to be enhanced in $\mathrm{Ca}$ relative to $\mathrm{Na}$ (Cohen 1974) where one may be observing gas that has been more recently shocked than the typical cloud. Nonmetallic species, such as C, N, O, Ar, S, P, and $\mathrm{Cl}$ should generally possess a uniform and relatively low depletion, characteristic of some possible membership in refractory grains. Recent observations (York 1977) appear to support this prediction.

Finally we remark that various parameters which are related to the location of the depleted species in the periodic table appear to correlate equally well with depletion-e.g., condensation temperature (Field 1974), first ionization potential (Snow 1975), polarizability or hydride recombination energy (Barlow 1974), and adsorption energy. One of the principal arguments of the present paper is that a more stringent test of depletion theories may come from understanding the apparent correlation between depletion of a particular species and cloud velocity.

We acknowledge helpful discussions with Drs. M. Jura, L. Spitzer, and D. York. The research of J. Silk has been supported in part by NASA grant NGR 05-003-578 and by NSF grant AST75-02181.

\section{REFERENCES}

Aannestad, P. A. 1972, unpublished Ph.D. thesis, University of California, Berkeley.

- 1973, Ap.J.Suppl., 25, 223.

Barlow, M. J. 1974, paper presented at Symposium on Solid State Astrophysics, Cardiff, Wales.

- 1975, unpublished D.Phil. thesis, University of Sussex. 1977, in preparation.

Barlow, M. J., and Silk, J. 1976, Bull. A AS, 8, 336.
Bohlin, R. C. 1975, Ap.J., 200, 402.

Burke, J. R., and Silk, J. 1974, Ap.J., 190, 1.

Cohen, J. G. 1974, Ap.J., 194, 37.

Field, G. B. 1974, Ap.J., 187, 453.

Gilra, P. P. 1972, Scientific Results from $O A O-2$, ed. A. Code

(NASA), p. 295.

Hobbs, L. M. 1976, Ap. J. (Letters), 206, L117. 
Ir. Jenkins, E. J., Silk, J., and Wallerstein, G. 1976, Ap. J. Suppl., : 32 , (in press).

Morton, D. C., and Hu, E. M. 1975, Ap. J., 202, 638.

, Shapiro, P. R., and Moore, R. T. 1976, Ap.J. (in press).

I Shull, J. M., Hobbs, L. M., and York, D. G. 1977, Ap. J. (Leiters)

InI (in press)

'I! Shull, J. M., and York, D. G. 1977, Ap. J. (in press).

Siluk, R. S., and Silk, J. 1974, Ap. J., 192, 51.

Snow, T. P. 1975, Ap.J. (Letlers), 202, 687.

Winters, H. F., and Sigmund, P. 1974, J. App. Phys., 45, 4760.

York, D. G. 1975, Ap.J. (Letters), 196, 2103.

- 1977, in preparation.

York, D. G., and Kinahan, G. F. 1976, preprint.

Michael J. Barlow: Joint Institute for Laboratory Astrophysics, University of Colorado, Boulder, CO 80309

JoSEPH SILx: Department of Astronomy, University of California, Berkeley, CA 94720 